\title{
Tmem45b is essential for inflammation and tissue injury-induced persistent mechanical hyperalgesia
}

\author{
Tadashi Tanioku \\ Wakayama Medical University School of Medicine \\ Masayoshi Nishibata \\ Wakayama Medical University School of Medicine \\ Yasuyuki Tokinaga \\ Wakayama Medical University School of Medicine \\ Kohtarou Konno \\ Hokkaido University \\ Masahiko Watanabe \\ Graduate School of Medicine, Hokkaido University \\ Hiroaki Hemmi
}

Okayama University of Science

\section{Yuri Ohta}

Wakayama Medical University School of Medicine

\section{Tsuneyasu Kaisho}

Wakayama Medical University School of Medicine

Tomoyuki Kawamata ( $\sim$ kawamata@wakayama-med.ac.jp)

Wakayama Medical University School of Medicine https://orcid.org/0000-0003-1535-6715

\section{Article}

Keywords: Tmem45b, peripheral nervous system, mechanical hyperalgesia

Posted Date: September 1st, 2021

DOI: https://doi.org/10.21203/rs.3.rs-829215/v1

License: (c) (1) This work is licensed under a Creative Commons Attribution 4.0 International License.

Read Full License 
Title: Tmem45b is essential for inflammation and tissue injury-induced persistent mechanical hyperalgesia

Tadashi Tanioku $^{1}$, Masayoshi Nishibata ${ }^{1}$, Yasuyuki Tokinaga ${ }^{1}$, Kohtaro Konno $^{2}$, Masahiko Watanabe ${ }^{2}$, Hiroaki Hemmi $^{3}$, Yuri Fukuda-Ohta ${ }^{3}$, Tsuneyasu Kaisho ${ }^{3}$, Tomoyuki Kawamata ${ }^{{ }^{*}}$

1. Department of Anesthesiology, Wakayama Medical University School of Medicine, Wakayama, Japan

2. Department of Anatomy, Hokkaido University Graduate School of Medicine, Hokkaido, Japan

3. Department of Immunology, Institute of Advanced Medicine, Wakayama Medical University School of Medicine, Wakayama, Japan

*Corresponding author: Tomoyuki Kawamata (kawamata@wakayama-med.ac.jp) 


\section{Acknowledgements}

We thank T. Ozasa at Department of Immunology, Institute of Advanced Medicine, Wakayama Medical University School of Medicine for generating KO mice. This study was supported by grants from JSPS KAKENHI Grant Numbers JP17H04322 (to T.Kaw.) and JP18K16460 (to T.T).

\section{Author contributions}

T.T. performed all experiments and analyzed data. Y.T. performed immunoblotting. M.N. and K.K. performed FISH and immunohistochemistry. M.W. generated an anti-Tmem45b antibody. T.Kai., H.H., and Y.F.-O. generated Tmem $45 \mathrm{~b}$ gene-deficient mice. T.Kaw. designed the experiments and wrote the manuscript. M.W., T.Kai. and H.H. reviewed the manuscript.

\section{Competing interests}

The authors declare no competing financial interests. 


\begin{abstract}
Persistent mechanical hyperalgesia, associated with peripheral inflammation and tissue injury, impairs patient's quality of life and daily activity. However, its molecular mechanism and treatment are yet to be deciphered. Herein, we report that Tmeme45b is expressed in a subset of unmyelinated primary sensory neurons and plays an essential role in inflammation and tissue injury-induced mechanical hyperalgesia. Our findings provide new insights into the mechanisms and the treatment of mechanical hyperalgesia.
\end{abstract}

\title{
Main
}

Pain functions as a warning system to alert us to real or impending injury and trigger appropriate protective responses. In addition, pain is not just a passive consequence of the transfer of peripheral input to the brain but also an active process. Inflammation, tissue injury, and nerve injury can induce a decrease in the pain threshold and an increase in the pain intensity, so-called hyperalgesia ${ }^{1}$. Persistent hyperalgesia causes distress to humans beyond the warning signals, interfering with physical and mental activities. Therefore, persistent hyperalgesia is an important clinical problem to be solved. Hyperalgesia can be divided into two types according to the modality of stimuli: thermal hyperalgesia and mechanical hyperalgesia. Recent advances in neuroscience research have revealed molecules such as TRP channels responsible for thermal hyperalgesia ${ }^{2}$. On the other hand, mechanisms responsible for mechanical hyperalgesia in mammals remain poorly understood ${ }^{3}$. Herein, we discuss a molecule that is crucial for mechanical hyperalgesia observed in inflammation and tissue injury in mice.

Nociceptive stimuli are sensed and transmitted by small myelinated sensory neurons and unmyelinated sensory neurons. Unmyelinated sensory neurons can be divided into two classes by isolectin B4 (IB4)binding $^{4,5}$ : IB4-not binding (IB4- ${ }^{-}$neurons, which are peptidergic and mainly express TRPV1 or CGRP and IB4-binding $\left(\mathrm{IB}^{+}{ }^{+}\right)$neurons, which are non-peptidergic ${ }^{6,7}$. Recent ablation studies have demonstrated that TRPV1- or CGRP-positive neurons are involved in heat pain and heat hyperalgesia but not in mechanical 
pain $^{8,9}$. However, the kind of pain IB4 ${ }^{+}$neurons are involved in remains unknown. To examine the role of IB4 ${ }^{+}$ neurons in pain transmission, we selectively ablated $\mathrm{IB}^{+}$neurons using IB4-saporin ${ }^{10-11}$. Intrasciatic nerve injection of IB4-saporin eliminated IB4 ${ }^{+}$afferents but not CGRP-positive afferents (Fig. 1a, b), while injection of saporin alone did not (data not shown). Ablation of $\mathrm{IB}^{+}{ }^{+}$neurons had no effect on the withdrawal latency to noxious heat stimuli and the withdrawal threshold to von Frey filaments (Fig. 1c), confirming previous finding ${ }^{11}$. Next, we examined the role of IB $4^{+}$neurons in three pain models with different neural mechanisms including a complete Freund's adjuvant (CFA)-induced inflammatory pain model, a skin incision-induced tissue injury pain model, and a peripheral nerve injury-induced neuropathic pain model. CFA injection and skin incision to the hindpaw induced a decrease in the withdrawal threshold to mechanical stimuli of von Frey filaments (mechanical hyperalgesia) and a decrease in the withdrawal latency to noxious heat stimuli (thermal hyperalgesia) in mice that received saproin (Fig. 1d, e). On the other hand, mice that received IB4-saporin showed thermal hyperalgesia but not mechanical hyperalgesia (Fig. 1d, e). The neuropathic pain model that we used shows mechanical hyperalgesia but not thermal hyperalgesia ${ }^{12}$. Both mice that received saporin and mice that received IB4-saporin also showed mechanical hyperalgesia but not thermal hyperalgesia (Fig. 1f), indicating that IB4 ${ }^{+}$neurons are not involved in mechanical hyperalgesia in a neuropathic pain model. Taken together, the results show that IB $4^{+}$neurons are selectively involved in inflammation and tissue injury-induced mechanical hyperalgesia, leading to the idea that molecules responsible for inflammation and tissue injuryinduced mechanical hyperalgesia could be expressed in IB4 $4^{+}$neurons.

To explore molecules responsible for inflammation and tissue injury-induced mechanical hyperalgesia, we compared gene expression profiles of dorsal root ganglia (DRGs) from mice that received either IB4-saporin or saporin using a cDNA array. Genes with an important difference in expression ( $>1.5$-fold change) were selected, and 43 were identified as being predominantly expressed in IB4 ${ }^{+}$neurons (Fig. 2a). Since Mishra et al. ${ }^{13}$ reported 153 genes selectively expressed in somatosensory neurons, we focused on 12 genes that overlapped between our 43 identified genes and the 153 somatosensory-specific genes (Fig. 2b). Among the 
12 genes, we decided to analyze Tmem $45 \mathrm{~b}$, for which the function in the nervous system has not yet been reported.

Real-time quantitative PCR analysis revealed that Tmem $45 b$ was mainly expressed in DRGs, aorta, bladder, and digestive tracts (Fig. 2c). Tmem $45 b$ was rarely expressed in the spinal cord and supra-spinal regions. As expected, double staining by in situ hybridization and immunohistochemistry revealed that most of the IB $4^{+}$ neurons $(91.8 \% \pm 1.8 \%, \mathrm{n}=4)$ were positive for Tmem $45 b$ mRNA in DRGs (Fig. 2d). Next, we examined Tmem45b expression using a specific antibody, validated by negative labeling in Tmem $45 b$-knockout DRGs and co-labeling for Tmem $45 b$ mRNA in wild-type DRGs (Extended Data Fig. 1). Immunohistochemical analysis showed that the percentage of Tmem45b-positive neurons among the total DRG neurons was 38.1\% $\pm 1.5 \%$ and that Tmem $45 \mathrm{~b}$ was selectively expressed in small-sized DRG neurons with an area $<600 \mu \mathrm{m}^{2}$ and rarely expressed in medium to large-sized DRG neurons (Fig. 2e, f). Double staining with several neuronal makers showed that Tmem45b was expressed in most IB4 $4^{+}$neurons $(93.5 \pm 2.4 \%)$, some TRPV1-positive neurons $(19.1 \pm 1.1 \%)$, and some CGRP-positive neurons $(7.1 \pm 1.3 \%)$, but was rarely expressed in NF200positive neurons $(0.1 \pm 0.1 \%$ ), which are considered to be myelinated sensory neurons (Fig. $2 \mathrm{~g}$ and Supplementary Table 3). Next, we examined the central and peripheral projections of Tmem45-positive afferents. In the spinal cord, Tmem45b-positive central afferents mainly terminated in the dorsal part of lamina II inner, which comprises IB4 ${ }^{+}$afferents (Fig. $2 \mathrm{~h}$ and see Methods). A retrograde tracing experiment using Fast blue showed that $28.1 \%$ of DRG neurons innervating glabrous skin in the hindpaw and $35.9 \%$ of DRG neurons innervating the tibialis anterior muscle were Tmem45b-positive, whereas the percentage of Tmem $45 \mathrm{~b}$ positive DRG innervating visceral organs (e.g., distal colon and bladder) was considerably low (Extended Data Fig. 2).

To clarify the role of Tmem45b in pain perception, we studied Tmem $45 b$ knockout mice (KO mice) (Fig. 3a and Extended Data Fig. 3). The KO mice were viable and did not show any overt motor defects or reflex 
impairments (data not shown). The KO mice showed withdrawal latency to noxious heat stimuli and withdrawal threshold to von Frey filaments that were comparable to those of WT mice (Fig. 3b). We examined the contribution of Tmem $45 \mathrm{~b}$ to mechanical hyperalgesia in an inflammatory pain model using KO mice. CFA injection to the hindpaw resulted in long-lasting mechanical and thermal hyperalgesia in WT mice, whereas KO mice showed thermal hyperalgesia but not mechanical hyperalgesia throughout the observation period (Fig. 3c). In addition, CFA injection-induced paw edema was comparable in WT and KO mice, suggesting that Tmem $45 \mathrm{~b}$ is not involved in the development of local inflammation (Extended Data Fig. 4). To exclude the possibility of secondarily developmental influence by gene knockout, we conducted a knockdown experiment using siRNA. We confirmed that our siRNA against Tmem $45 b$ reduced Tmem45b mRNA in Lewis lung carcinoma cells to $\sim 20 \%$ compared with scrambled siRNA (Extended Data Fig. 5). First, siRNA against Tmem $45 b$ was intrathecally administered before CFA injection (pre-treatment). Intrathecal injection of siRNA against Tmem $45 b$ or scrambled siRNA once a day for 3 consecutive days did not change withdrawal latency to noxious heat stimuli and withdrawal threshold to von Frey filaments compared with those before intrathecal administration (Fig. 3d upper panel). Twenty-four hours after the 3rd siRNA administration, CFA was injected into the hindpaw. The mice that received siRNA against Tmem $45 b$ developed thermal hyperalgesia, but mechanical hyperalgesia was significantly prevented compared to that that received scrambled siRNA (Fig. $3 \mathrm{~d}$, upper). In addition, effects of siRNA against Tmem $45 b$ on established hyperalgesia after CFA injection (post-treatment) were examined (Fig. 3d, lower). Since CFA-induced hyperalgesia was maintained from 4 to 
$168 \mathrm{hr}$ after CFA injection, siRNA was administered at 24, 48, and $72 \mathrm{hr}$ after CFA injection. Intrathecal injection of siRNA against Tmem $45 \mathrm{~b}$ partially improved mechanical hyperalgesia compared to injection of scrambled siRNA. In both pre- and post-treatment with siRNA, siRNA against Tmem $45 b$ significantly decreased Tmem $45 b$ mRNA levels in DRGs compared with scrambled siRNA (Fig. 3e). These results suggest that inhibition of CFA-induced mechanical hyperalgesia observed in $\mathrm{KO}$ mice is not due to secondarily developmental change of the nervous system induced by downregulation of Tmem $45 \mathrm{~b}$ but is due to direct involvement of Tmem $45 \mathrm{~b}$ in the development and maintenance of CFA-induced mechanical hyperalgesia.

Finally, we examined the involvement of Tmem $45 \mathrm{~b}$ in hyperalgesia observed in different pain models including the skin incision-induced tissue injury pain model and the peripheral nerve injury-induced neuropathic pain model. Although WT mice showed skin incision-induced thermal and mechanical hyperalgesia, KO mice showed thermal hyperalgesia but not mechanical hyperalgesia, similar to the results obtained in the CFA-induced inflammation pain model (Fig. 3f). In the neuropathic pain model, nerve injuryinduced hyperalgesia was comparable in WT mice and $\mathrm{KO}$ mice (Fig. $3 \mathrm{~g}$ ), indicating that Tmem45b is not involved in nerve injury-induced hyperalgesia.

It has been reported that Tmem $45 \mathrm{~b}$ is involved in the development of several types of cancer ${ }^{14-17}$. In this study, we demonstrated for the first time that Tmem $45 \mathrm{~b}$ does not play a role in sensing noxious mechanical stimuli under normal conditions but is essential for inflammation and tissue injury-induced mechanical hyperalgesia. The molecular mechanisms controlled by Tmem $45 \mathrm{~b}$ in inflammation or tissue injury-induced 
mechanical hyperalgesia remain to be elucidated. Although an in silico study has suggested that Tmem $45 \mathrm{~b}$ is a7-transmembrane protein ${ }^{18}$, Tmem $45 \mathrm{~b}$ does not have any typical amino acid sequences of $\mathrm{G}$ protein-coupled receptors (GPCRs) such as the CWxP motif in TM6, NPxxY motif in TM7, or D/ERY motif in TM3 ${ }^{19}$. This suggests that Tmem $45 \mathrm{~b}$ does not belong to the family of GPCRs. Further studies will be necessary to clarify the molecular function of Tmem $45 \mathrm{~b}$ and the mechanisms of Tmem $45 \mathrm{~b}$-induced mechanical hyperalgesia. A therapeutic approach targeting Tmem $45 \mathrm{~b}$ is attractive for two reasons. One reason is that it does not affect physiological pain sensitivity, and it can therefore relieve pathological pain while retaining pain as a warning signal. The other reason is that Tmem $45 \mathrm{~b}$ is mainly expressed in peripheral neurons and is rarely expressed in central neurons. Therefore, such a therapeutic approach may avoid the possible side effects associated with the central nervous system including addiction, which is known to lead to serious health concerns in opioid therapy, the so-called "opioid crisis"20. 


\section{References}

1. Treede, R.D,. et al. Peripheral and central mechanisms of cutaneous yperalgesia. Prog Neurobiol 38, 397421, (1992)

2. Vriens, J., Nilius ,B. \& Voets, T. Peripheral thermosensation in mammals. Nat. Rev. Neurosci. 15, 573$589(2014)$

3. Hill, R.Z. \& Bautista, D.M. Getting in touch with mechanical pain mechanisms. Trends in Neurosci. 43, $311-325(2020)$

4. Silverman, J.D. \& Kruger, L. Selective neuronal glycoconjugate expression in sensory and autonomic ganglia: relation of lectin reactivity to peptide and enzyme markers. J Neurocytol. 19, 789-801 (2020)

5. Nagy, J.I. \& Hunt, S.P. Fluoride-resistant acid phosphatase-containing neurones in dorsal root ganglia are separate from those containing substance P or somatostatin. Neuroscience 7, 89-97 (1982)

6. Zwick, M., et al. Glial cell line-derived neurotrophic factor is a survival factor for isolectin B4-positive, but not vanilloid receptor 1-positive, neurons in the mouse. J Neurosci. 22, 4057-4065 (2002)

7. Woodbury, C.J., et al. Nociceptors lacking TRPV1 and TRPV2 have normal heat responses. J Neurosci. 24, 6410-6415 (2004)

8. McCoy, E.S., et al. Peptidergic CGRP $\alpha$ primary sensory neurons encode heat and itch and tonically suppress sensitivity to cold. Neuron $\mathbf{8}, 138-151$ (2013)

9. Cavanaugh, D.J., et al. Distinct subsets of unmyelinated primary sensory fibers mediate behavioral responses to noxious thermal and mechanical stimuli. Proc. Natl. Acand. Sci. 106, 9075-9080 (2009)

10. Ono, K., et al. TRPV1 expression level in isolectin $\mathrm{B}_{4}$-positive neurons contributes to mouse strain difference in cutaneous thermal nociceptive sensitivity. J. Neurophysiol. 113, 3345-3355 (2015)

11. Pinto, L.G., et al. Non-peptidergic nociceptive neurons are essential for mechanical inflammatory hypersensitivity in mice. Mol. Neurobiol. 56, 5715-5728 (2019) 
12. Holmes, F.E., et al. Transgenic overexpression of galanin in the dorsal root ganglion modulates painrelated behaviors. Proc. Natl. Acand. Sci. 100, 6180-6185 (2013)

13. Mishra, S.K., Holzman, S. \& Hoon, M.A. A nociceptive signaling role for neuromedin B. J. Neurosci.32, $8686-8695$ (2012)

14. Shen, K., et al. Knockdown of TMEM45B inhibits cell proliferation and invasion in gastric cancer. Biomed. Pharmacother. 104, 576-81 (2018)

15. Li, Y., et al. Silencing transmembrane protein 45B (TNEM45B) inhibits proliferation, invasion, and tumorigenesis in osteosarcoma cells. Oncol. Res. 25, 1021-1026 (2017)

16. Hu, R., et al. TMEM45B, up-regulated in human lung cancer, enhances tumorigenicity of lung cancer cells. Tumor Biol. 37, 12181-12191 (2016)

17. Zhao, L.C., et al. TMEM45B promotes proliferation, invasion and migration and inhibits apoptosis in pancreatic cancer cells. Mol. Biosyst. 12, 1860-1870 (2016)

18. Wrzesinski, T., et al. Expression of pre-selected TMEMs with predicted ER localization as potential classifiers of ccRCC tumors. BMC. Cancer 15, 518 (2015)

19. Filipek, S. Molecular switches in GPCRs. Curr. Opin. Struct. Biol. 55, 114-120 (2019)

20. Volkow, N.D. \& Collins, F.S.C. The role of science in addressing the opioid crisis. $N$ Engl J Med. 377, 391-394 (2017) 


\section{Methods}

\section{Animals}

Approval was obtained from the Wakayama Medical University Animal Care and Use Committee for all the procedures of this study that were in accordance with the ethical guidelines of the National Institutes of Health and of the International Association for the study of Pain. Adult male C57BL/6N mice (20-25 g, Japan SLC, Japan) and Tmem 45b-deficient mice of C57BL/6N strain (20-25 g) were used for experiments. The mice were housed in a temperature-controlled $\left(21^{\circ} \mathrm{C} \pm 1^{\circ} \mathrm{C}\right)$ room under a $12 \mathrm{~h}$ light/dark cycle and were given food and water ad libitum. Behavioral tests were conducted at 7-12 weeks-of-age.

\section{Deletion of IB4 positive afferents in sciatic nerve}

Using 3\% sevoflurane, B6 mice were anesthetized. Then, to expose the sciatic nerve, a small incision was made on the skin of the leg proximal to the knee and the skin and underlying muscle opened by blunt dissection. A needle of 29.5 gauge was attached to a $10 \mu 1$ syringe (Hamilton, Reno, NV, USA), and $1 \mu 1$ of IB4-saporin $(1 \mu \mathrm{g} / \mu \mathrm{l}$ saline; Advanced Targeting Systems, San Diego, CA, USA) or saporin ( $1 \mu \mathrm{g} / \mu 1$ saline $)$ were injected as control into the left sciatic nerve. The muscle tissue was closed back over the nerves and then skin was sutured shut using 6-0 nylon sutures. Any animals displaying motor impairment during recovery were immediately euthanized. Animals without any motor impairment were used for the experiments 7-10 days after intrasciatic injection. After the behavioral experiment, the deletion of IB4 positive afferents was checked 
by IB4 binding in dorsal horn of the L4-5 spinal cord.

\section{Generation of Tmem $45 \mathrm{~b} \mathrm{KO}$ mice}

We generated Tmem $45 b$ knockout mice by using CRISPR/Cas9 technology. The guide RNAs (gRNAs) for targeting Tmem $45 b$ were designed by Invitrogen ${ }^{\mathrm{TM}}$ GeneArt ${ }^{\mathrm{TM}}$ CRISPR Search and Design Tool (Thermo Fisher Scientific, Waltham, MA, USA) and synthesized using GeneArt ${ }^{\mathrm{TM}}$ Precision gRNA Synthesis Kit (Thermo Fisher Scientific) (Supplementary Table 1). Cas9 endonuclease mRNA was generated via in vitro transcription using MEGAshortscript T7 (Thermo Fisher Scientific) and purified using MEGAclear kit (Thermo Fisher Scientific). One cell-stage fertilized embryos from B6C3F1 females mated with C57BL/6N males were injected with the gRNAs and Cas 9 mRNA and transferred to the oviducts of pseudopregnant foster mice. Founders bearing deletion of Tmem $45 b$ were identified by PCR with the primers, Tmem $45 \mathrm{~b}-1$ and Tmem45b-2, which should yield a 4282- and 1181-bp product from the wild-type and mutated alleles (Supplementary Table 2). Mutant mice were backcrossed to C57BL/6N mice.

\section{Knockdown of Tmem45b}

\section{Preparation of siRNA against Tmem45b and scrambled siRNA}

siRNA against mice Tmem $45 b$ were purchased from Sigma Aldrich (St. Louis, MO, USA). Sense and antisense siRNA sequences were: 5'-CUUAUGUGCUCCUAGGGCU-3' and 5'-AGCCCUAGGACACAUAAG-3'. A 
scrambled control siRNA without significant homology to any known mouse gene sequence was obtained from

Sigma Aldrich. For in vivo siRNA transfer, the hemagglutinating virus of the Japan (HVJ) envelope vector system (HVJ Envelope Vector Kit GenomONE-Neo; Ishihara Sangyo Kabushikigaisya, Ltd., Osaka, Japan) was used. siRNA was incorporated into the HVJ-envelope vector as per the manufacturer's instructions, as reported previously ${ }^{21}$. In brief, the mixture was centrifuged $\left(10,000 \mathrm{~g}, 10 \mathrm{~min}, 4^{\circ} \mathrm{C}\right)$ and the pellet was suspended in $10 \mu \mathrm{l}$ of buffer solution after mixing $40 \mu \mathrm{l}$ (1 assay unit) of the HVJ-envelope vector with $4 \mu \mathrm{l}$ of the enclosing factor. Then, $10 \mu \mathrm{l}$ siRNA solution was added, and the mixture was kept on ice for 5 min. The final concentration of siRNA was $5 \mu \mathrm{g} / 10 \mu \mathrm{l}$. The knockdown efficacy of siRNA used in this study was examined using Lewis lung carcinoma cells, which expressed Tmem45b.

\section{Implantation of a chronic intrathecal catheter for siRNA administration}

A chronic intrathecal (i.t.) catheter was implanted into the intrathecal space, as reported previously ${ }^{22}$. In brief, a polyurethane i.t. catheter with an inner diameter of $0.35 \mathrm{~mm}$ and an outer diameter of $0.84 \mathrm{~mm}$ (R-ITC; Neuroscience Inc., Tokyo, Japan) was inserted $5 \mathrm{~mm}$ cephalad into the mouse lumbar subarachnoid space at the L4/L5 intervertebral space, with the tip of the catheter located near the lumbar enlargement of the spinal cord, under general anesthesia with 3\% sevoflurane in 100\% oxygen via a nose cone. The catheter was tunneled subcutaneously and externalized through the skin in the neck region. The volume of dead space of the intrathecal catheter was $3 \mu 1$. The effects of i.t. lidocaine $(2 \%, 2 \mu 1)$ were examined one day after implantation of the catheter. Animals that had shown complete paralysis of the tail and bilateral hind legs after i.t. lidocaine 
were exclusively used in the following experiments. Every day, for three days after surgery, 10,000 units/kg of penicillin was injected subcutaneously to prevent infection. Following the surgeries, mice that showed paralysis or impaired motor function were excluded from this study. In 21 of the 22 mice, the catheter implantation procedure was performed successfully.

\section{Knockdown of Tmem45b in dorsal root ganglions}

Using a microinjection syringe (Hamilton) connected to an i.t. catheter under brief general anesthesia (sevoflurane in oxygen), administration of i.t. siRNA was accomplished. Over a 10-s period with a single injection volume of $5 \mu \mathrm{g} / 10 \mu \mathrm{l}$ followed by a flush of physiological saline $(3 \mu \mathrm{l})$, siRNA against Tmem $45 \mathrm{~b}$ or scrambled siRNA was manually administered. Mice fully recovered within 2 min after discontinuance of general anesthesia. For 3 consecutive days, injections were given daily.

\section{Pain models}

We used the following mouse model of pain:

\section{Inflammatory pain}

Mice were lightly anesthetized using inhaled 3\% sevoflurane and then were injected into the plantar surface of the left hind paw, with $20 \mu \mathrm{L}$ of complete Freund's adjuvant (CFA) (Sigma Aldrich) ${ }^{23}$.

\section{Skin incision-induced tissue injury pain}

Using inhaled 3\% sevoflurane, mice were lightly anesthetized. A plantar incision was made according to a 
modification of a previous report ${ }^{24}$. Through skin and fascia of the plantar surface of the foot, a 5-mm longitudinal incision was made with a number 11 scalpel blade. Started $2 \mathrm{~mm}$ from the proximal edge of the heel, the incision was extended distally. Using curved forceps, the underlying muscle was elevated, which left the muscle origin and insertion intact. The skin was sutured with a 7-0 nylon mattress suture and then covered with antibiotic ointment after wound hemostasis.

\section{Peripheral nerve injury-induced neuropathic pain}

Surgery was performed as previously described ${ }^{25}$. Using inhaled $3 \%$ sevoflurane, mice were lightly anesthetized. The left hind limb was shaved using trimmers and then sterilized using betadine and ethanol. To expose the three branches of the sciatic nerve, a small incision was made on the skin of the leg proximal to the knee and the skin and underlying muscle opened by blunt dissection. Tightly ligated with 6-0 nylon sutures, the peroneal and sural branches were transected below the ligature, and a 2-3-mm section distal to the ligature was removed. The tibial nerve was carefully avoided and left intact. The muscle tissue was closed back over the nerves and then skin was sutured shut using 6-0 nylon sutures.

\section{Behavioral Tests}

\section{Mechanical test}

On a wire mesh table for $1 \mathrm{hr}$ the day before and $30 \mathrm{~min}$ prior to testing, mice were habituated to opaque Plexiglas chambers. Using a set of calibrated Touch Test Sensory Evaluator (North Coast Medical, Morgan 
Hill, CA, USA) following the Up-Down method, testing was performed ${ }^{26}$. For each mouse beginning with a $0.16 \mathrm{~g}$ filament, the $50 \%$ paw withdrawal threshold was determined. Between the walking pads for $3 \mathrm{~s}$ or until a response such as a sharp withdrawal, shaking, or licking of the limb was observed, each filament was applied to the plantar surface of the hind paw. Incidents of rearing or normal ambulation during filament application were not counted. In between each application until the thresholds were determined, testing was performed with a 5-min interval.

\section{Thermal test}

According to a previous study, withdrawal latency to noxious heat stimuli was measured ${ }^{27}$. To the test chamber for $1 \mathrm{hr}$ the day before and $30 \mathrm{~min}$ the day of testing, animals were placed in an acrylic chamber on a glass top table and then allowed to acclimate. A radiant heat source of constant intensity, using a Plantar Analgesia Meter (IITC Life Sciences, Woodland Hills, CA, USA), was focused on the plantar surface of the hind paw and the latency to paw withdrawal measured. Upon paw withdrawal or after $20 \mathrm{~s}$ of exposure to prevent injury, the heat source was shut off. With a 5-min interval between tests, the test was repeated thrice and the results for each paw were averaged together.

\section{Evaluation of Paw Edema}

By a $40 \mu \mathrm{L}$ intraplantar injection of CFA, paw edema was induced in the hind paw of the mice. By measuring the volume of hind paw using a digital plethysmometer (Ugo Basile) under general anesthesia with 3\% 
sevoflurane, the edema was monitored. The results are expressed in milliliters.

\section{Generation of Tmem45b antibody}

We developed antibody to mouse Tmem45b by immunizing synthetic 264-278 amino acid peptide (GenBank \# NM_144936.1) conjugated with keyhole limpet hemocyanin (KLH). In the first immunization and incomplete adjuvant, Tmem45b/KLH was emulsified with Freund's complete adjuvant (DIFCO) and then injected subcutaneously into a Japanese White Rabbit. Using the Tmem $45 \mathrm{~b}$ peptide coupled to CNBr-activated Sepharose 4B (GE Healthcare, IL, USA), serum was collected and affinity-purified after the sixth injection.

\section{Immunohistochemistry}

Mice were anesthetized using inhaled 3\% sevoflurane and then perfused transcardially with phosphatebuffered saline (PBS), followed by 4\% paraformaldehyde (PFA) in 0.1-M PB for DRG immunohistochemistry and by $3 \%$ glyoxal working solution $(7.8 \mathrm{~mL}$ of $40 \%$ Glyoxal, $0.75 \mathrm{~mL}$ of glacial acetic acid, and $91.425 \mathrm{~mL}$ of $\mathrm{ddH}_{2} \mathrm{O}, \mathrm{pH} 4.0$ ) for spinal cord immunohistochemistry. The spinal cord and dorsal root ganglia were harvested during a laminectomy. Tissues were post-fixed in 4\% PFA or 3\% glyoxal working solution for 2-6 hr, cryoprotected in 30\% sucrose in PBS overnight, and then cut with a cryostat (Leica CM3050S, Leica, Wetzlar, Germany) into $16 \mu \mathrm{m}$ sections for DRGs and $50 \mu \mathrm{m}$ for spinal cord; these were then placed directly onto silane coated slides (Muto) or into wells containing PBS. Slices were blocked with 10\% normal donkey 
serum in PBS $+0.2 \%$ triton-X (PBS-T) for $30 \mathrm{~min}$ at room temperature (RT) and then incubated in primary

antibody diluted in PBS-T overnight at RT for fluorescent immunostaining. Then, sections were washed in

PBS-T and incubated in AlexaFluor ${ }^{\mathrm{TM}}$ secondary antibodies (Jackson ImmunoResearch, West Grove, PA,

USA) diluted 1:200 in PBS-T for $2 \mathrm{hr}$ at RT. Then, slices were washed in PBS-T and cover-slipped with

Vectashield $^{\mathrm{TM}}$ (VECTOR laboratories, Burlingame, CA, USA). Photographs were taken using confocal laser

scanning microscopes (ECLIPSE C1, NIKON, Tokyo, Japan) For immunofluorescence staining at the

following dilutions, the following primary antibodies were used: anti-NF200 raised in mouse (1:2000; Sigma

N0142), anti-CGRP raised in guinea pig ( $1 \mu \mathrm{g} / \mathrm{ml}$; Frontier Institute CGRP-GP-Af280), anti-TRPV1 raised in

guinea pig $(1 \mu \mathrm{g} / \mathrm{ml})^{28}$, biotinylated IB4 (1:100; Sigma L3759), anti-PGP9.5 raised in mouse (1:100;

Neuromics MO25010), and anti-Tmem45b raised in rabbit $(0.1 \mu \mathrm{g} / \mathrm{ml})$. In spinal immunohistochemistry,

TRPV1 and IB4 were used to label lamina I/outer part of lamina II and the dorsal part of lamina II inner, respectively ${ }^{29,30}$.

\section{Retrograde labeling}

Using Fast Blue (FB; 1\% in PBS, Polysciences GmbH, Germany), retrograde labeling of sensory neurons innervating the hind paw skin, tibialis anterior muscle, distal colon, and bladder was performed in B6 mice.

Mice were anesthetized with 3\% sevoflurane, and FB $(10 \mu \mathrm{l})$ was injected in hind paw $(\mathrm{n}=4)$ and tibialis anterior muscle $(\mathrm{n}=4)$. Injections were administered with a $10 \mu 1$ syringe (Hamilton) and 29.5-gauge needle. 
Moreover, injection to visceral organs was administered as previously described ${ }^{31}$. In brief, mice were anesthetized with $3 \%$ sevoflurane, and to reveal the visceral organs, a midline laparotomy was performed. FB was injected into the wall of distal colon $(n=4,6$ injections per animal, total volume $2 \mu \mathrm{l})$ and the wall of bladder $(n=4$, one injection per animal, $10 \mu \mathrm{l})$. The ventral membrane was closed and the skin was sutured shut using 6-0 nylon sutures. Animals were anesthetized by inhalation of 3\% sevoflurane 7-10 days after FB injection and perfused transcardially using saline $(0.9 \% \mathrm{NaCl})$, followed by $4 \%$ PFA in $0.1-\mathrm{M} \mathrm{PB}$. Dissection of lumbar (L4-L5) DRG, lumbar (L3-L4) DRG, thoracolumbar (T13), and lumbosacral (S1) DRG for analysis of innervation to hind paw, tibialis anterior muscle, distal colon, and bladder, respectively, was performed. Six slices were dissected from one DRG and used for immunohistochemistry.

\section{Fluorescence in situ hybridization}

As previously described, fluorescence in situ hybridization was done ${ }^{32}$. To detect Tmem45b mRNA, digoxigenin (DIG) labeled cRNA probes were prepared. cDNA fragments of Tmem45b (90 -926; National Center for Biotechnology Information Reference Sequence NM_144936.1) were subcloned into the Bluescript II plasmid vector and sequenced. As described previously, preparation of cRNA probes was performed ${ }^{33}$. The sequence of primers used for the creation of cRNA probes is listed in Supplementary Table 2. In vitro transcription was performed using T7 or T3 RNA polymerase using the linearized plasmid. Using DIG labeling kits (Roche Diagnostic GmbH, Mannheim, Germany), probes were synthesized. Sections were treated with 
the following incubation steps: acetylation with $0.25 \%$ acetic anhydride in $0.1 \mathrm{M}$ triethanolamine- $\mathrm{HCl}, \mathrm{pH} 8.0$, for $10 \mathrm{~min}$, and pre-hybridization for $1 \mathrm{~h}$ in hybridization buffer $(50 \%$ formamide, $50 \mathrm{mM}$ Tris- $\mathrm{HCl}, 0.02 \%$ Ficoll, $0.02 \%$ polyvinylpyrrolidone, $0.02 \%$ bovine serum albumin, $0.6 \mathrm{M} \mathrm{NaCl}, 200 \mathrm{~g} / \mathrm{ml}$ tRNA, $1 \mathrm{mM}$ EDTA, and $10 \%$ dextran sulfate, $\mathrm{pH} 7.5$ ). Hybridization was performed at $63.5^{\circ} \mathrm{C}$ for $12 \mathrm{~h}$ in hybridization buffer supplemented with cRNA probes at a dilution of $1: 1000$. At $61^{\circ} \mathrm{C}$, post-hybridization washing was done successively with $5 \mathrm{X}$ SSC for $30 \mathrm{~min}$, $4 \mathrm{X}$ SSC containing $50 \%$ formamide for $30 \mathrm{~min}$, 2X SSC containing $50 \%$ formamide for $30 \mathrm{~min}$, and $0.1 \mathrm{X} \mathrm{SSC}$ for $30 \mathrm{~min}$. Sections were incubated at RT in NTE buffer $(0.5 \mathrm{M}$ $\mathrm{NaCl}, 0.01 \mathrm{M}$ Tris- $\mathrm{HCl}$, and $5 \mathrm{mM}$ EDTA, $\mathrm{pH}$ 7.5,) for $20 \mathrm{~min}, 20 \mathrm{mM}$ iodoacetamide in NTE buffer for 20 min, and TNT buffer (0.1 M Tris-HCl, $0.15 \mathrm{M} \mathrm{NaCl}, \mathrm{pH}$ 7.5) for $5 \mathrm{~min}$. Sections were incubated with DIG blocking solution (TNT buffer containing 1\% blocking reagent [Roche Diagnostic GmbH] and 4\% normal sheep serum) for $30 \mathrm{~min}$ and $0.5 \%$ tryamide signal amplification (TSA) blocking reagent (Thermo Fisher, Waltham, MA, USA) in TNT buffer for 30 min for immunohistochemical detection of DIG. Using the Cy3TSA plus amplification kit (Thermo Fisher), fluorescence detection was done. Using peroxidase-conjugated anti-digoxigenin antibody (1:1000, $1 \mathrm{~h}$; Invitrogen, Carlsbad, CA, USA), the detection was performed. Finally, sections were stained with IB4 (1:100; Sigma L3759).

\section{Quantitative analysis for immunohistochemistry}

For quantitative analysis of DRG neurons, images obtained from confocal laser scanning microscopes were 
imported into the ImageJ software (NIH, Bethesda, MD). A positive cell was defined as a cell with an intensity two times greater than that of the background. Tissues were counterstained for a pan neuronal marker, PGP9.5, to visualize and measure the cross-sectional area of a neuronal cell body. Only neurons with clearly visible nuclei were counted. Quantification of date may have yielded biased estimates of actual numbers of cells and neurons because a stereological approach was not used in this study. Sections that were at least $48 \mu \mathrm{m}$ apart were counted for each DRG to prevent duplicate counting of neuronal cell bodies.

\section{Western blot analysis}

Frozen tissues were cut into small pieces and homogenized in ice-cold lysis buffer (50 mM HEPES, $1 \%$ Triton X-100, $50 \mathrm{mM} \mathrm{NaCl}, 50 \mathrm{mM}$ sodium fluoride, $5 \mathrm{mM}$ EDTA, $10 \mathrm{mM}$ sodium pyrophosphate, $1 \mathrm{mM}$ phenylmethanesulfonyl fluoride, $1 \mathrm{mM} \mathrm{Na}_{3} \mathrm{VO}_{4}, 10 \mu \mathrm{g} / \mathrm{mL}$ leupeptin and $20 \mu \mathrm{g} / \mathrm{mL}$ aprotinin, $\mathrm{pH}$ 7.5, $)^{34}$. For $15 \mathrm{~min}$ at $4^{\circ} \mathrm{C}$, tissue homogenates were centrifuged at 15,000 g. Using the bicinchoninic acid method, the supernatant was collected and the protein concentration was determined ${ }^{35}$. By mixing them with an equal volume of $2 \times$ SDS sample buffer, samples of the protein extracts were prepared. For use at a later date, the diluted samples were boiled for $3 \mathrm{~min}$ and stored at $-80^{\circ} \mathrm{C}$. Aliquots for each sample (equivalent total protein content, $40 \mu \mathrm{g}$ ) were used in each experiment. Proteins were separated using 10\% sodium-dodecyl-sulfate polyacrylamide gel electrophoresis (SDS-PAGE) and then electroblotted onto nitrocellulose membranes. The electroblotted membranes were incubated in blocking buffer (containing $20 \mathrm{mM}$ Tris, $150 \mathrm{mM} \mathrm{NaCl}, 3 \%$ 
bovine serum albumin and $0.02 \%$ sodium azide, $\mathrm{pH} 7.5$ ) overnight at $4{ }^{\circ} \mathrm{C}$ and then incubated with a rabbit anti-Tmem45b antibody $(0.1 \mu \mathrm{g} / \mathrm{ml})$, and a rabbit antibeta-actin antibody (1:2000; Cell Signaling 4970S). After washing again, the membrane was incubated with a secondary antibody, antirabbit IgG antibody (1:2000; Cell Signaling 7074S), for detection of Tmem45b or beta-actin. Using the enhanced chemiluminescence (ECL) system (Amersham Pharmacia Biotech, Piscataway, NJ, USA), the densities of the immunoreactive Tmem45b bands and beta-actin bands were detected

\section{Real time quantitative PCR}

All tissues were freshly isolated from adult C57BL/6N mice. The total RNA samples were extracted using the RNeasy Mini Kit (Qiagen, Hilden, Germany). Using the PrimeScript RT Master Mix (Takara, Japan), the first strand cDNA was synthesized. The templates were amplified using TB Green Fast qPCR Mix (Takara, Japan). Experiments were carried out in triplicates using the LightCycler480 (Roche Diagnostic GmbH). The product calibrations and normalizations were performed using the 2- $\Delta$ cycle threshold $(\mathrm{Ct})$ method $(\mathrm{Schmittgen}$ and Livac, 2008), where $\Delta \mathrm{Ct}=(\mathrm{Ct}$ [target gene] $-\mathrm{Ct}$ [reference gene] $)$. For the analysis of mRNA expression in different tissues, Gapdh was used as a reference gene. The sequence of the primers used in quantitative PCR is listed in Supplementary Table 2.

\section{Gene profiling}


Using RNeasy Mini Kit (Qiagen, Germany) including a DNA digestion step, RNA from DRG dissected IB4saporin or saporin injected mice $(\mathrm{n}=3$ mice per condition) was isolated. Small aliquot from each sample was subjected to a quality control test using the RNA 6000 Pico/Nano LabChip technology (Agilent Technologies, Santa Clara, CA, USA) prior to being used for a microarray. For microarray, only RNA with sharp and distinct $28 \mathrm{~S}$ and $18 \mathrm{~S}$ ribosomal RNA peaks and ratio $28 \mathrm{~S} / 18 \mathrm{~S}>1.7$ or RIN $>7$ was further processed ${ }^{36}$. According to the Affymetrix standard protocol, cRNA double T7 linear amplification was performed for each sample. A total of amplified and biotinylated cRNA was then processed on Affymetrix GeneChip ${ }^{\mathrm{TM}}$ Mouse Gene 2.0 ST Array and signal values and detection calls (present or absent) for all transcripts were assigned with GeneChip $^{\mathrm{TM}}$ Command Console (TAC4.0, Affymetrix). Using the Robust Multi-array Average algorithm, Affymetrix CEL files were normalized for Bioinformatics analysis. Fold differences were calculated as the ratio between the average values within each condition. We defined a criterion of a 1.5 -fold and greater difference plus fold discovery rate adjusted $p$-value of $<0.1$ to identify transcripts that are differentially expressed between the controls and the ablated mice.

\section{Statistical analysis}

Statistical analyses were performed in this study using JMP ${ }^{\mathrm{TM}}$ statistical software (version 14.2; SAS institute,

Cary NC, USA). We have expressed quantitative data as mean \pm s.d. No statistical methods were used to predetermine sample sizes, but our sample sizes were selected on the basis of previously published studies (see 
Reporting Summary for references). The allocation of mice to groups was done randomly in all experiments.

No animals or data points were excluded from the analyses. Prior to choosing the statistical test, normality tests and $F$ tests for equality of variances were performed. For two-group comparison, statistics were based on two-sided paired or unpaired Student's $t$ test or Mann-Whitney rank-sum tests. For multiple comparisons, oneway ANOVA followed by Dunnett's test or two-way ANOVA were performed, unless indicated otherwise. $P$ values $<0.05$ were considered as statistically significant. Statistical details of specific experiments-for instance, exact $n$ values and what $n$ represents, statistical tests used, and $P$ values have been summarized in the figure legends.

\section{References}

21. Yamamoto, K., et al. Mol. Pain 14, 1-11 (2018).

22. Furuse, S., et al. Anesthesiology 111, 173-186 (2009).

23. Honore, P, et al. Neuroscience 98, 585-598 (2000).

24. Banik, R.K., Woo, Y.C., Park, S.S. \& Brennan, T.J. Anesthesiology 105, 1246-1253 (2006).

25. Shields, S., Eckert, W.A. \& Basbaum, A.I. J. Pain 4, 465-470 (2003).

26. Chaplan, S.R., et al. J. Neurosci. Methods 53, 55-63 (1994).

27. Barabas, M.E., \& Stucky, C.L. Mol. Pain 9, 9 (2013).

28. Fuseya, S., et al. Anesthesiology 125, 204-218 (2016).

29. Zylka, M.J., Rice, F.L., \& Anderson, D.J. Neuron 45, 17-25 (2005). 
30. Cavanaugh, D.J., et al. J. Neurosci. 31, 10119-10127 (2011).

31. Prato, V., et al. Cell Rep. 11, 3102-3115 (2017).

32. Miyazaki, T., et al. Eur. J. Neurosci. 33, 82-94 (2010).

33. Yamasaki, M., et al. J. Neurosci. 21, 7691-7704 (2001).

34. Molloy, C.J., Taylor, D.S. \& Weber, H. J. Biol. Chem. 268, 7338-7345 (1993).

35. Smith, P.K., et al. Anal. Biochem. 50, 76-85 (1985).

36. Schroeder, A., et al. BMC Molecular. Biol. 7, 3 (2006).

37. Choi, S., et al. J. Physiol. Sci. 67, 431-438 (2017).

38. Martin, W.J., Cao, Y.Q. \& Basbaum, A.I. J Neurophysiol. 91, 1945-1954 (2004). 


\section{Figure 1}

a

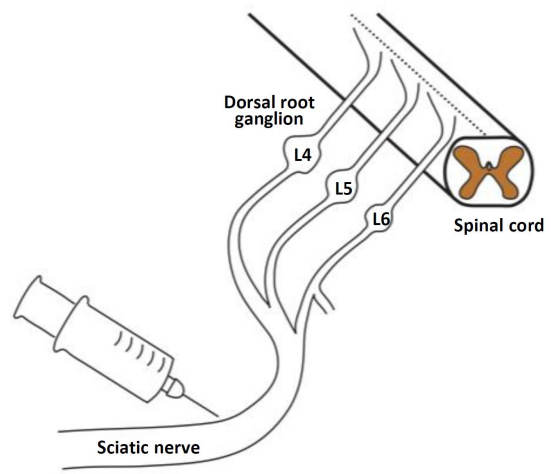

b

Ipsilateral side to Contralateral side IB4-saporin injection

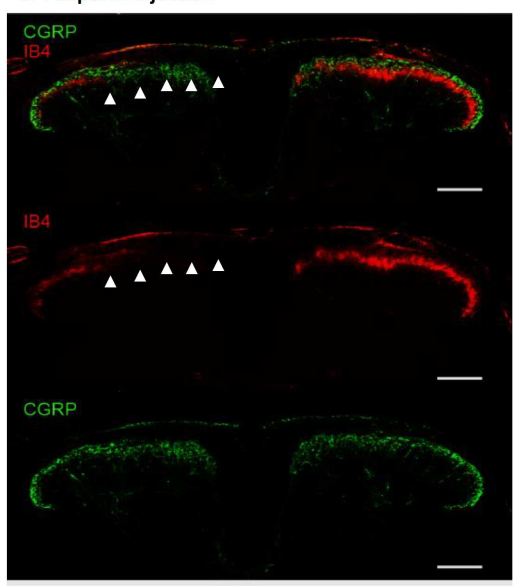

C
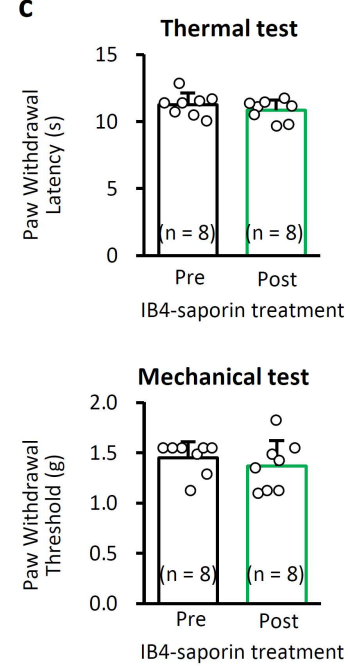

d
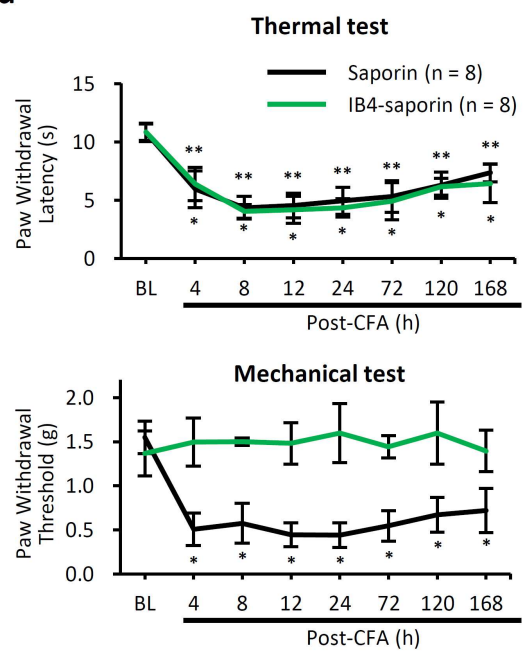
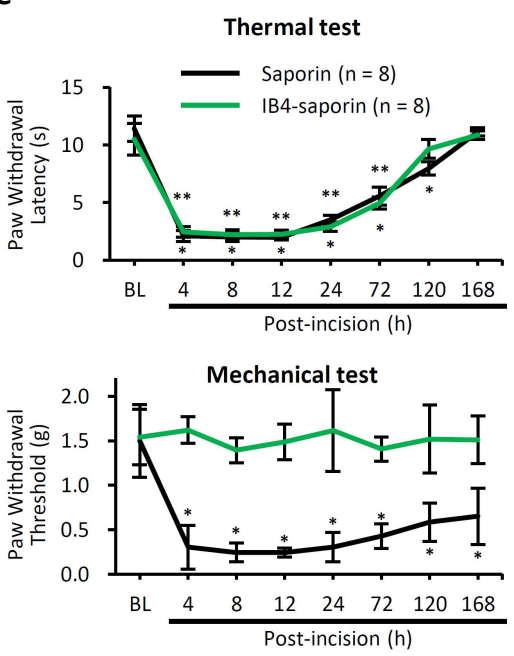

f

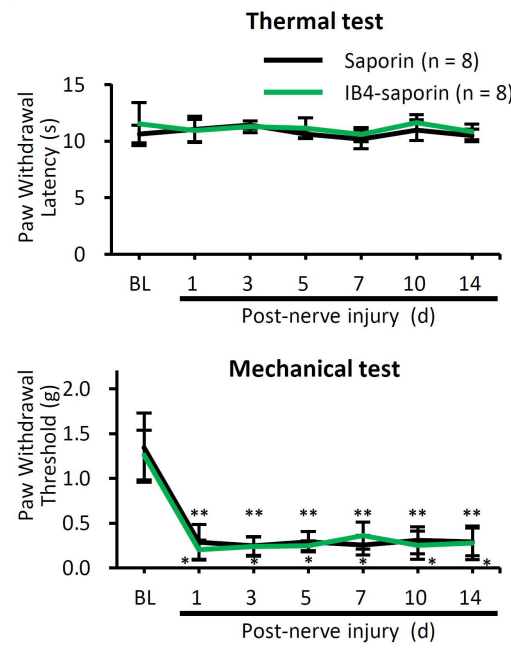


Fig. 1 IB4-binding neurons are critical for inflammation and tissue injury-induced mechanical hyperalgesia. a, Schematic of IB4-saporin or saporin injection into the left sciatic nerve. b, IB4-saporin selectively ablated IB4 ${ }^{+}$afferents (red) but not CGRP-positive afferents (green) in the dorsal horn of the spinal cord ipsilateral side to IB4-saporin injection. Immunostaining was performed 7 days after IB4-saporin injection. White arrowheads indicate elimination of IB4 ${ }^{+}$afferents. Scale bar: $200 \mu \mathrm{m}$. c, Ablation of I IB4 $4^{+}$ neurons had no effect on withdrawal latencies to noxious heat stimuli nor withdrawal thresholds to von Frey filaments (before IB4-saporin injection vs 7 days after IB4-saporin injection, $P=0.45$ for withdrawal latency, $P=0.62$ for withdrawal threshold; two-tailed paired Student's $t$-test). $\mathbf{d}-\mathbf{f}$, Effects of IB4-saporin treatment in three different pain models (d, CFA-induced inflammation pain model; e, skin incision-induced tissue injury pain model; f, peripheral nerve injury-induced neuropathic pain model). IB4-saporin treatment selectively inhibited mechanical hyperalgesia in inflammation pain model (d) and tissue injury pain model (e). In each figure, the upper panel shows the time courses of withdrawal latency to noxious heat stimuli and the lower panel the withdrawal threshold to von Frey filaments. BL: basal value, which is a value before CFA injection, skin incision, or nerve injury. Mice receiving saporin and mice receiving IB4-saporin in each pain model, $* P$ $<0.0001$ vs. BL within a group comparison of mice receiving saporin, $* * P<0.0001$ vs. BL within a group comparison of mice receiving IB4-saporin; one-way ANOVA followed by Dunnett's test. All data in Fig. 1 are presented as mean \pm s.d. 
Figure 2

a

\begin{tabular}{|c|c|c|c|c|c|}
\hline Fold Change & Gene Symbol & Acc number & Fold Change & Gene Symbol & Acc number \\
\hline-2.24 & Mrgprx1 & NM_207540 & -1.62 & A3galt2 & NM__001009819 \\
\hline-2.21 & Mrgprb5 & NM_207538 & -1.60 & Trpa1 & NM_177781 \\
\hline-2.09 & Mrgpra3 & NM_153067 & -1.59 & Kcnt1 & NM_175462 \\
\hline-2.05 & Chrna6 & NM_021369 & -1.59 & Dgki & NM_001081206 \\
\hline-1.93 & Mrgprd & NM_203490 & -1.58 & Gpr165 & NM_029536 \\
\hline-1.77 & Gm16364 & NR_152259 & -1.57 & Tmem45b & NM_144936 \\
\hline-1.75 & $\mathrm{Ctxn} 3$ & NM_001134697 & -1.57 & Kcnmb1 & NM_031169 \\
\hline-1.75 & Syt10 & NM_018803 & -1.57 & Moxd1 & NM_021509 \\
\hline-1.73 & Rasgrp1 & NM_011246 & -1.56 & Ano3 & NM_001128103 \\
\hline-1.72 & Hs6st2 & NM_001077202 & -1.55 & D130009118Rik & NR_015593 \\
\hline-1.71 & Gm7271 & NR_033501 & -1.55 & Gm16532 & NM_001134752 \\
\hline -1.70 & Lpar3 & NM_022983 & -1.54 & Fam188b2-ps & NM_001142781 \\
\hline-1.70 & Kcng3 & NM_153512 & -1.54 & Plds & NM_176916 \\
\hline-1.68 & Ldb2 & NM_010698 & -1.54 & Grik1 & NM_146072 \\
\hline-1.66 & Scn11a & NM_011887 & -1.53 & Paqrs & NM_028748 \\
\hline-1.66 & Cd55 & NM_010016 & -1.53 & Tmem255a & NM_172930 \\
\hline-1.65 & Trpe3 & NM_019510 & -1.52 & Prkcq & NM_008859 \\
\hline-1.65 & Icall & NM_001357296 & -1.52 & Olfr1000 & NM_001011695 \\
\hline-1.65 & Gm21889; Gm21920 & & -1.51 & Prkar2b & NM_011158 \\
\hline-1.64 & Mal2 & NM_178920 & -1.50 & Gna14 & NM_008137 \\
\hline-1.63 & Cypaf39 & NM_177307 & -1.50 & Slc35f4 & NM_029238 \\
\hline-1.62 & st6gal2 & NM_001347403 & & & \\
\hline
\end{tabular}

b

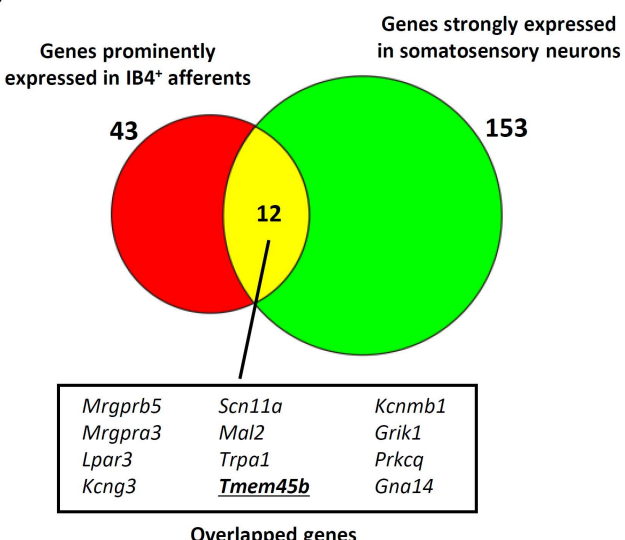

C

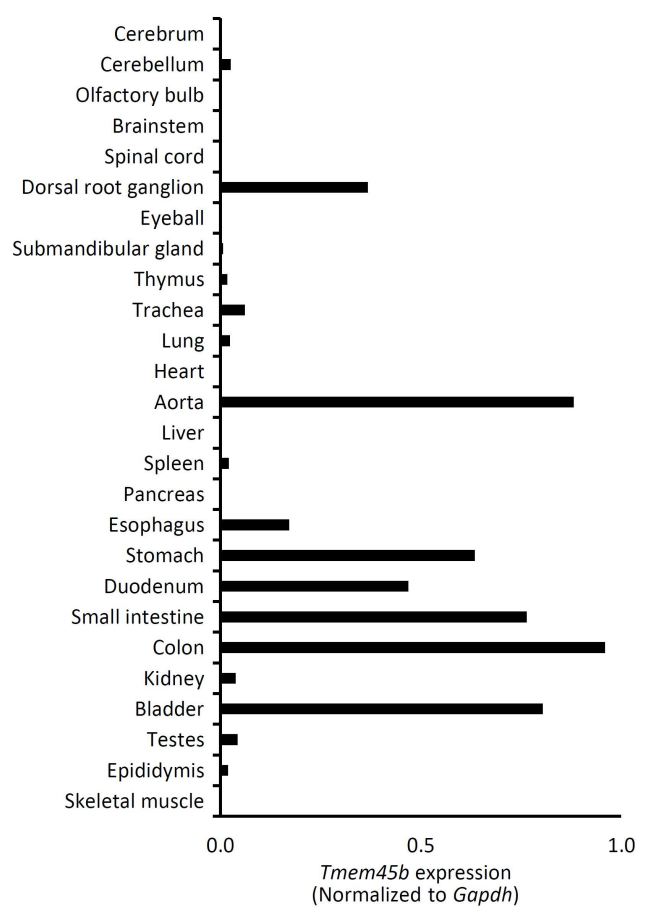

g

marker
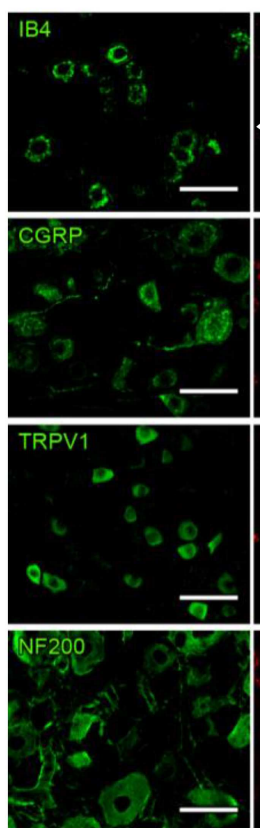

Tmem45b
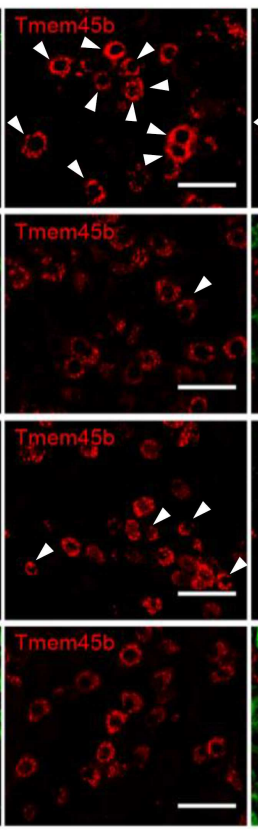

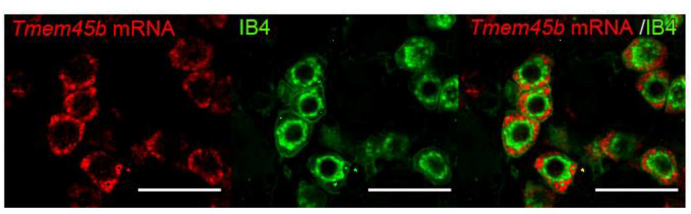

e

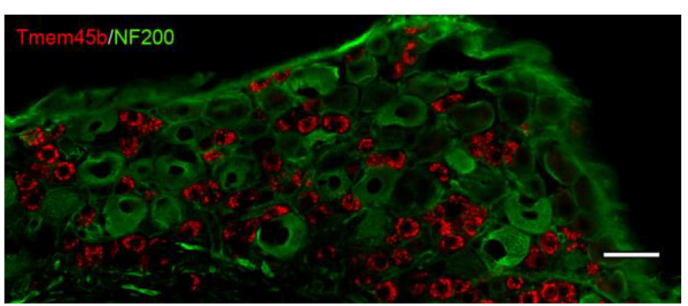

f

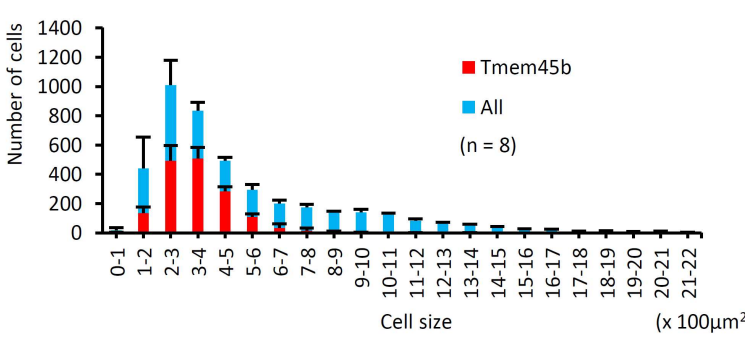

h
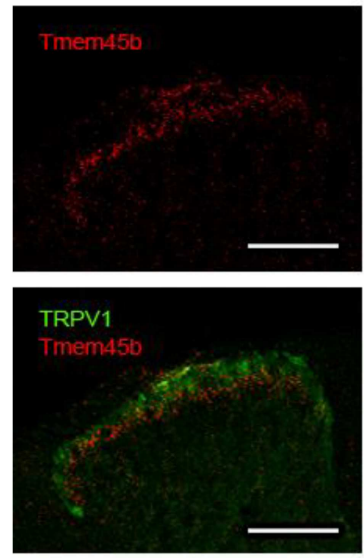

Tmem45b

184 
Fig. 2 Tmem 45b is expressed in a specific subset of small-sized primary sensory neurons. a, Genes identified by comparison of dorsal root ganglions between mice receiving saporin and mice receiving IB4saporin. Genes that vary $>1.5$-fold change (decrease) in mice receiving IB4-saproin compared with mice receiving saporin were selected to show predominent difference of expression $(n=3)$. $\mathbf{b}$, Schematic of selection of interested genes. The red circle and the green circle indicate 43 genes prominently expressed in $\mathrm{IB}_{4}^{+}$neurons and 153 genes selectively expressed in somatosensory neurons shown by Mishra et $\mathrm{al}^{14}$, respectively. The yellow region indicates 12 overlapped genes. c, Real time quantitative PCR analysis revealed that Tmem $45 b$ was mainly expressed in DRG, aorta, bladder, and digestive tracts in mouse tissues. Bars represent Tmem $45 b$ mRNA expression normalized to Gapdh. d, Expression of Tmem $45 b$ mRNA in IB4 ${ }^{+}$dorsal root ganglion (DRG) neurons. Tmem 45b mRNA (red) and IB4 (green) were stained in DRG by fluorescent in situ hybridization and immunohistochemistry, respectively. Scale bar:50 $\mu \mathrm{m}$. e, Representative image of Tmem $45 \mathrm{~b}$ (red) expression in DRG. NF200 (green), a marker of myelinated neurons. Scale bar:50 $\mu \mathrm{m}$. f, Cell size distribution of Tmem45b in L4/5 DRGs. $n=4$. $\mathbf{g}$, Colocalization of Tmem45b (red) with neuronal markers (green) including IB4 (nonpeptidergic unmyelinated neurons), CGRP (peptidergic neurons), TRPV1 (heat and capsaicin sensitive nociceptive neurons), and NF200 in DRGs. Arrowheads indicate double positive neurons. Data of colocalization were obtained from 4 mice. A scale bar represents $50 \mu \mathrm{m}$. h, Spinal innervation of Tmem45b positive afferent. red, Tmem45b; green, TRPV1; blue, IB4. Scale bar:200 $\mu \mathrm{m}$. All data in Fig. 2 are presented as mean \pm s.d. 


\section{Figure 3}

a

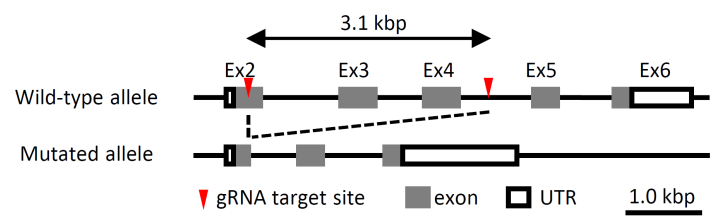

b
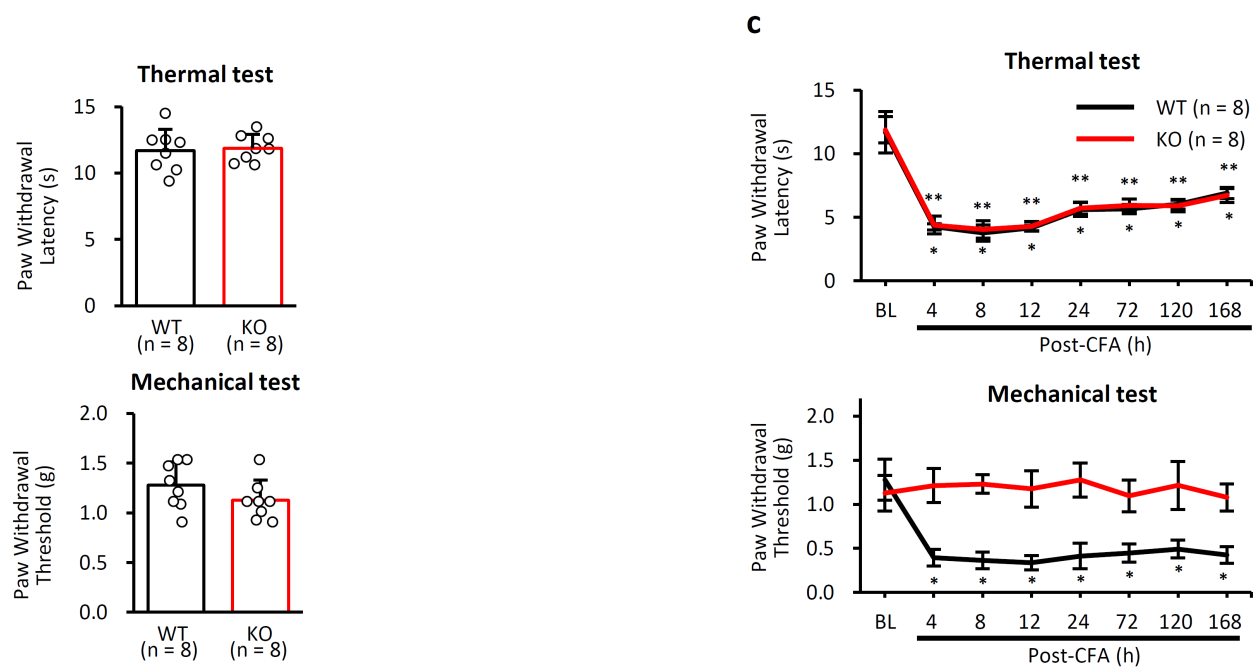

d

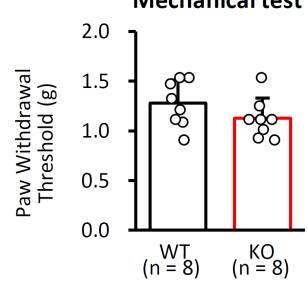

e

Pre-treatment
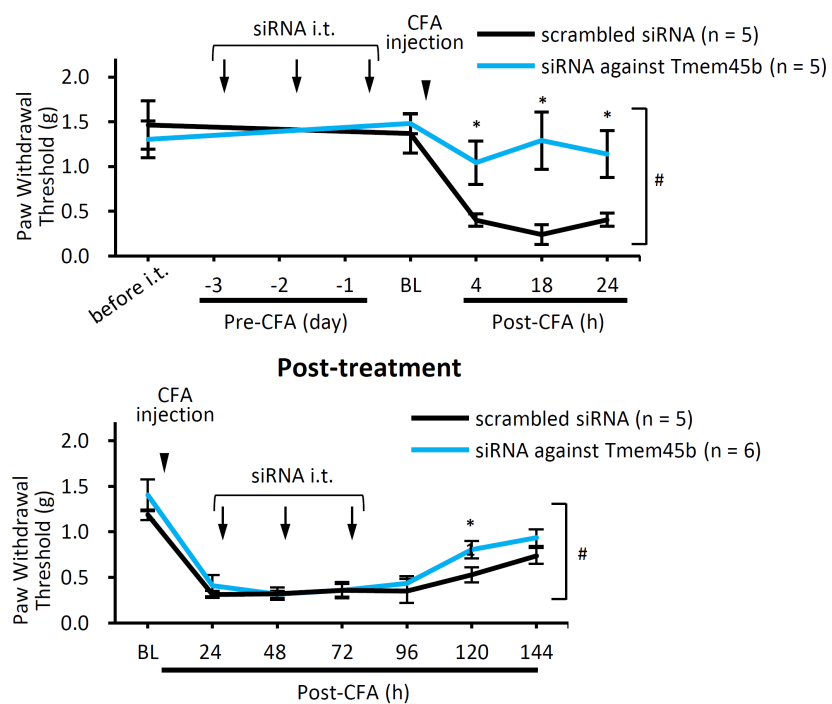

f
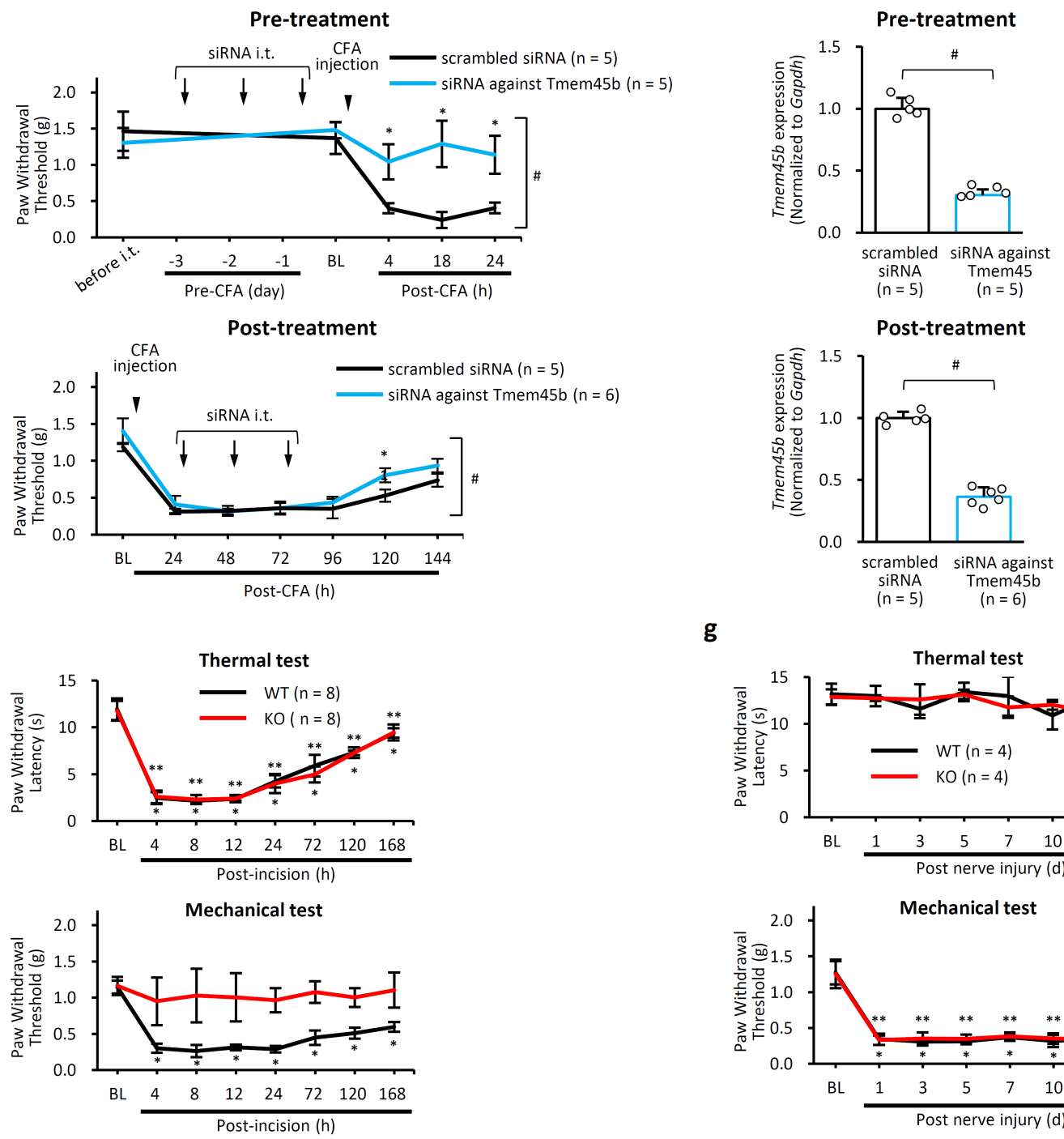

g
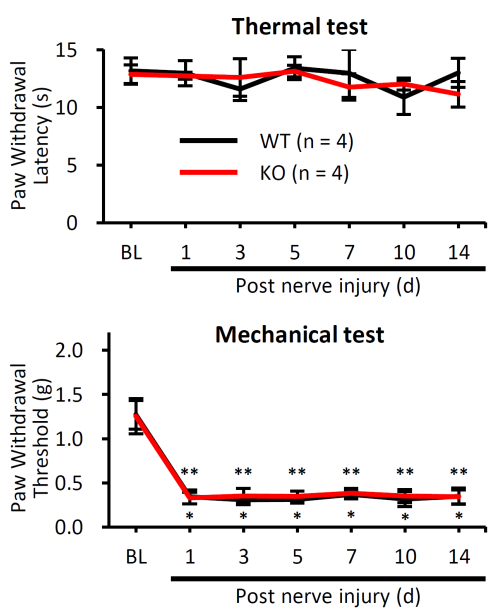
Fig. 3 Tmem 45b is critical for inflammation and tissue injury-induced mechanical hyperalgesia. a, Generation of Tmem $45 b$ knockout mice (KO mice). Targeting mediated by two gRNAs induced deletion of a $3.1 \mathrm{kbp}$ as indicated by red arrow heads. $\mathbf{b}$, Tmem $45 \mathrm{~b}$ gene knockout does not affect withdrawal latency to noxious heat stimuli (upper panel, $P=0.91$ ), or withdrawal threshold to von Frey filaments (lower panel, $\mathrm{n}=$ $8, P=0.39$ ); two-tailed unpaired Student's $t$-test. c, Effects of Tmem $45 b$ gene knockout on hyperalgesia in CFA-induced inflammation pain model. ${ }^{*} P<0.0001$ vs. BL within a group comparison of WT mice, ${ }^{* *} P<$ 0.0001 vs. BL within a group comparison of KO mice; one-way ANOVA followed by Dunnett's test. d, e, Effects of Tmem $45 b$ knockdown in CFA-induced inflammation pain model. d, Effects of pre- and posttreatment of siRNA on mechanical hyperalgesia. An upper panel and a lower panel show effects of pre- and post-treatment, respectively. SiRNA against Tmem $45 b(5 \mu \mathrm{g})$ or scrambled siRNA were administered 3 consecutive days before CFA injection (pre-treatment) or after CFA injection (post-treatment). Arrows indicate siRNA administration. Arrowheads indicate CFA injection. ${ }^{\sharp} P<0.0001$, siRNA against Tmem $45 b$ vs scramble; two-way ANOVA. ${ }^{*} P<0.0002$ between group comparisons at each time point; two-tailed unpaired Student's $t$-test for analysis of pretreatment. ${ }^{\#} P<0.004$, siRNA vs scramble; two-way ANOVA. ${ }^{*} P<0.0002$ between group comparisons at each time point; two-tailed unpaired Student's $t$-test for analysis of post-treatment. "before i.t." means a value before siRNA injection. e, Effects of siRNA on Tmem $45 b$ mRNA level in DRGs. L4/5/6 DRG samples were collected after the last behavioral assessment in both cases. A bar shows mean of relative expression level of Tmem $45 b$ mRNA normalized to Gapdh. Number of mice was the same as in Fig. 3d. ${ }^{\#} P<0.0001$, siRNA against Tmem $45 b$ vs scrambled RNA; two-tailed unpaired Student's $t$-test. f, g, Effects of Tmem $45 \mathrm{~b}$ gene knockout on hyperalgesia in skin incision-induced tissue injury pain model (f) and neuropathic pain model (g). ${ }^{*} P<0.0001$ vs. BL within a group comparison of WT mice, ${ }^{* *} P<0.0001 \mathrm{vs.} \mathrm{BL}$ within a group comparison of KO mice; one-way ANOVA followed by Dunnett's test. All data in Fig. 3 are presented as mean \pm s.d. BL: basal value, corresponding to the value before CFA injection, skin incision, or nerve injury in Fig. 3. 

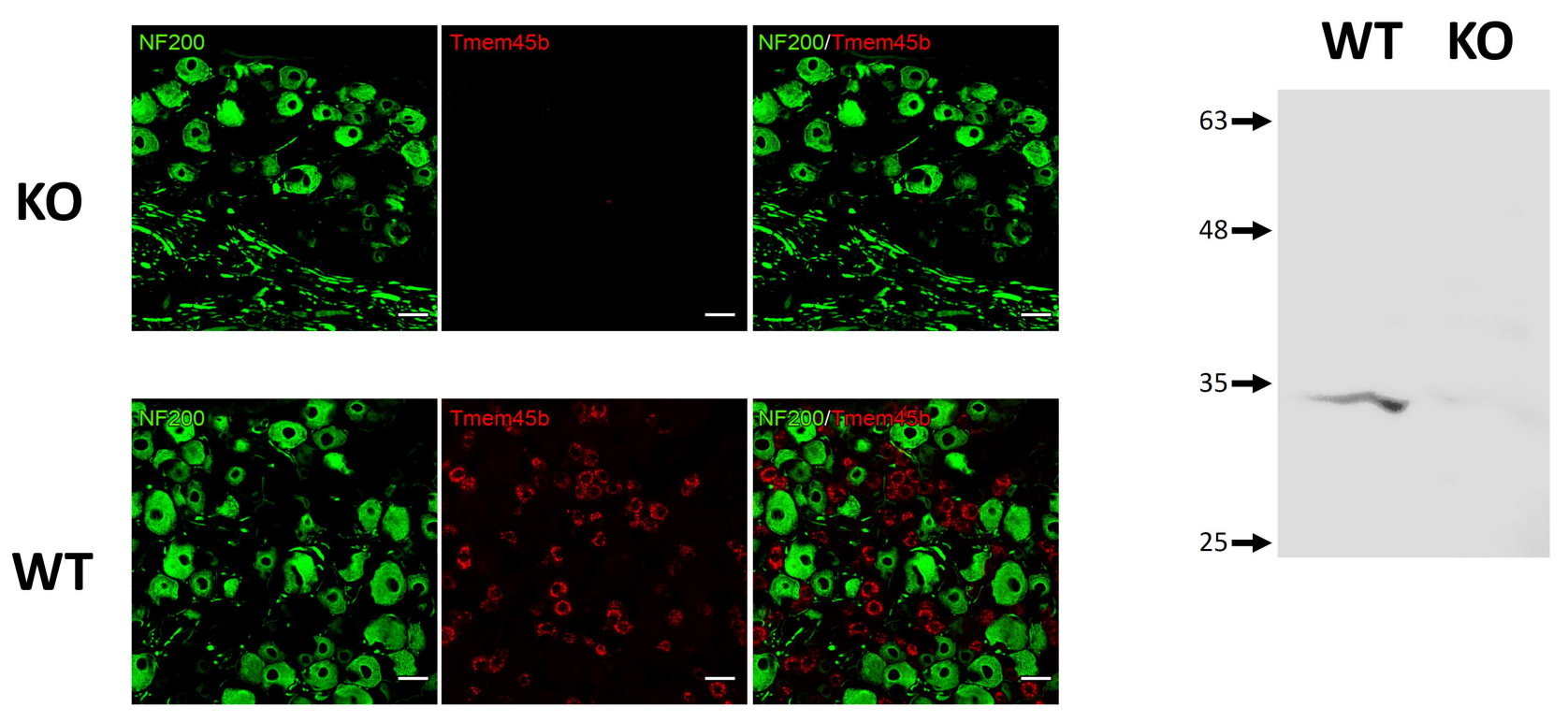

C

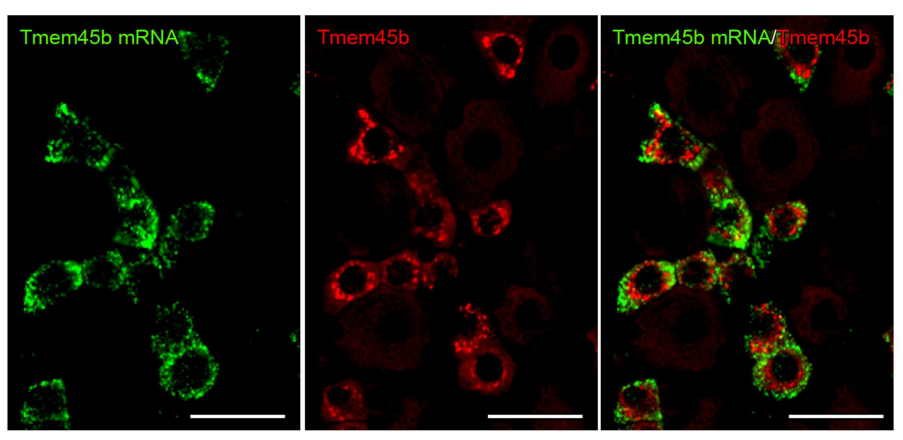

Extended Data Fig. 1 Specificity of the Tmem45b antibody. a, Immunostaining of DRG sections from WT and $\mathrm{KO}$ mice. A subpopulation of small-diameter DRG neurons from WT, but not Tmem45b KO, was recognized by our antibody. (Red, Tmem45b immunoreactivity; green, NF200 immunoreactivity). b, Western blot of DRG sections from WT and KO mice. Tmem45b antibody recognized a major band at around $33 \mathrm{kDa}$ in the DRG of WT mice. On the other hand, no immunoreactivity was found in the DRG of KO mice. c, Double staining with fluorescence in situ hybridization and immunohistochemistry. The antibody selectively recognized Tmem $45 b$ mRNA positive neurons (Red, Tmem45b immunoreactivity; green, Tmnem $45 b$ mRNA). The results demonstrate that the Tmem $45 \mathrm{~b}$ antibody we generated specifically detects Tmem $45 \mathrm{~b}$. Scale bar: $50 \mu \mathrm{m}$. 


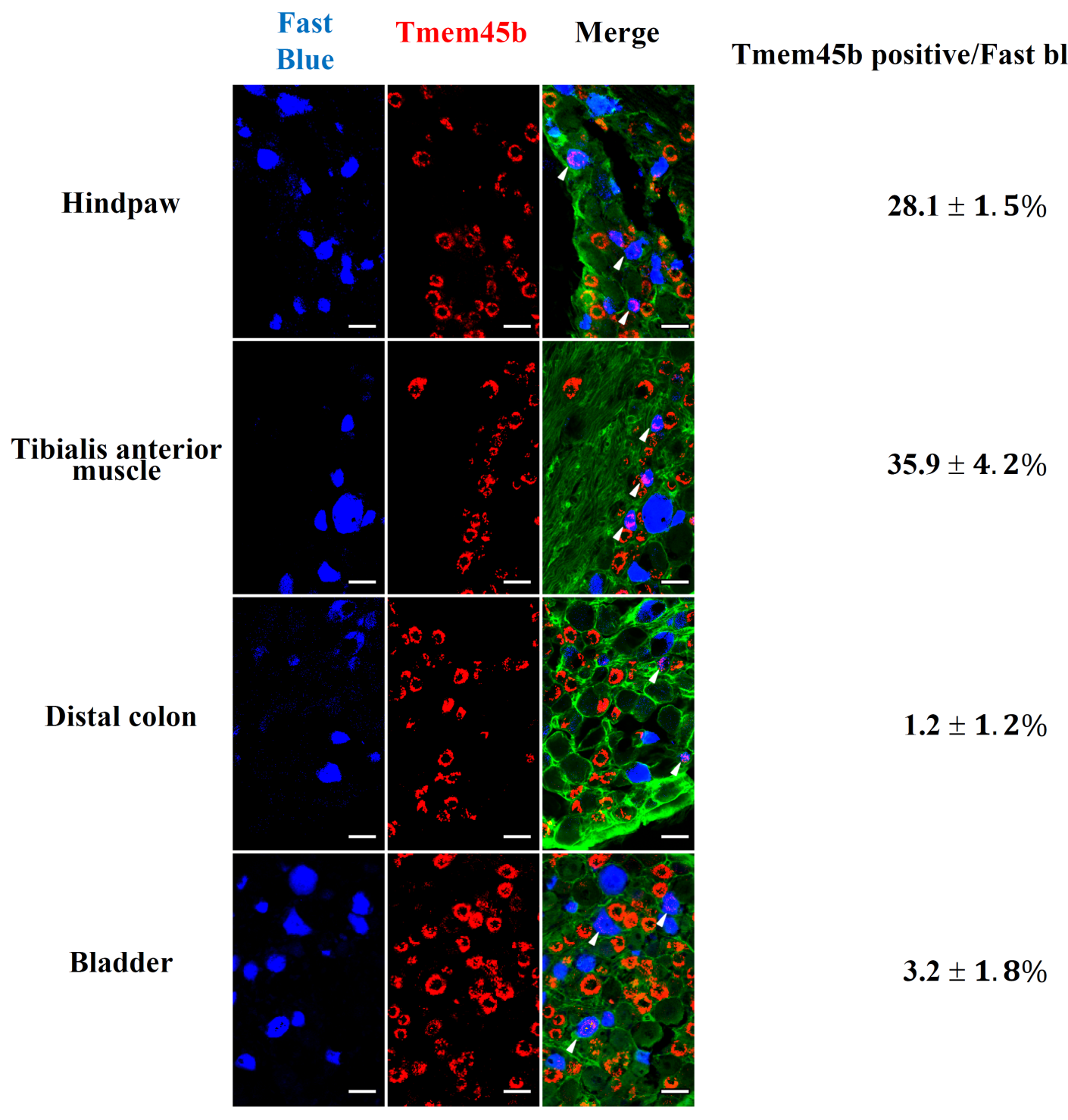

Extended Data Fig. 2 Innervation of Tmem45b positive afferents to distinct tissues. The number of Tmem45b and FB double positive neuron/FB positive neuron were $171.8 \pm 27.0 / 612.0 \pm 86.4$ in hindpaw (n $=4), 128.3 \pm 38.5 / 352.0 \pm 75.3$ in tibialis anterior muscle $(n=4), 5.3 \pm 5.2 / 379.3 \pm 76.3$ in distal colon $(n=$ 4), and $22.3 \pm 12.1 / 672.0 \pm 53.7$ in bladder $(n=4)$, respectively. Blue, fast blue; red, Tmem45b positive immunoreactivity; green, PGP9.5 positive immunoreactivity. White arrowheads indicate double positive neurons. Scale bar: $50 \mu \mathrm{m}$. 


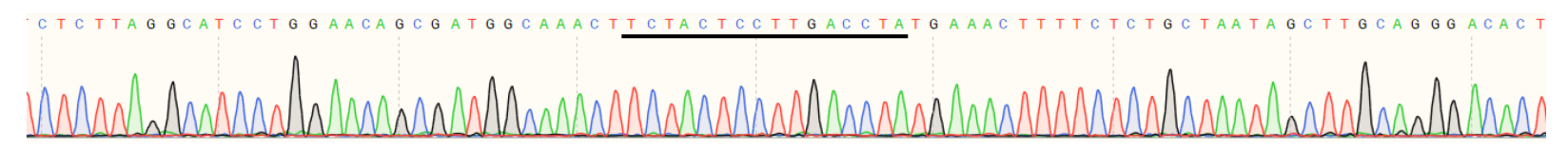

b

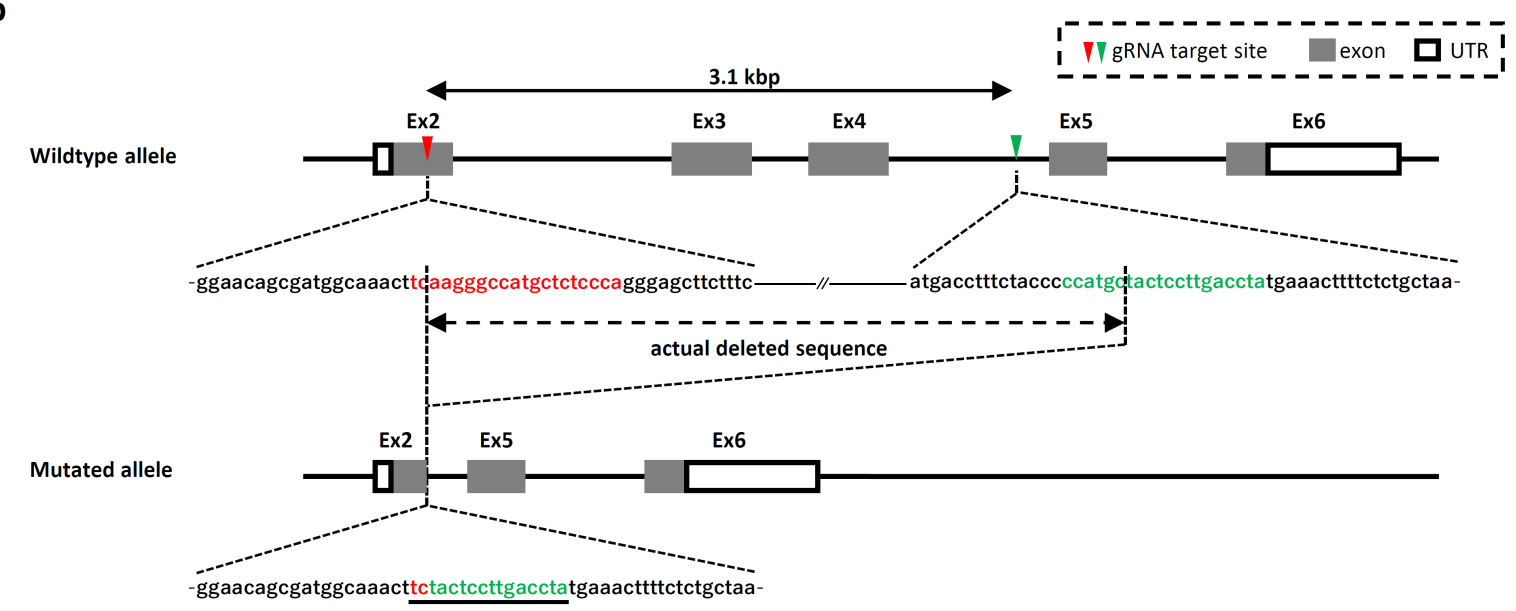

C

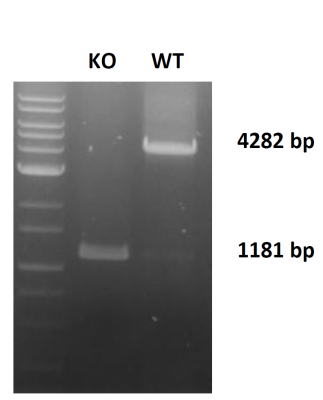

d

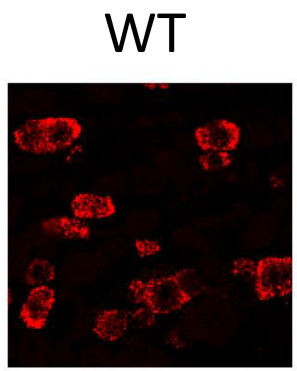

$\mathrm{KO}$

Extended Data Fig. 3 Generation of Tmem $45 b$ KO mice. a, Sequence chromatograms of Tmem $45 b$ KO mice. An underline shows the actual base sequence. $\mathbf{b}$, Schematic illustration of deleted sequence. Exon2 contains the translational initiation codon. In an upper panel, the gRNAs were designed for the sequence labeled in red in exon 2 and for the sequence labeled in green the intron between exons 4 and 5 in wildtype allele. A dashed bi-directional arrow indicates deleted sequence determined from chromatogram. A lower panel shows mutated allele. c, The electrophoresis of genotyping of WT mice and Tmem $45 \mathrm{~b}$ KO mice using tail sample. The PCR product of Tmem $45 b \mathrm{KO}$ mice is about $3.1 \mathrm{kbp}$ shorter than that of WT mice as shown in supplementary figure 3a and 3b. d, Fluorescence in situ hybridization in DRG sections from WT and KO mice. Tmem45 mRNA was not detected in DRG of KO mice. 


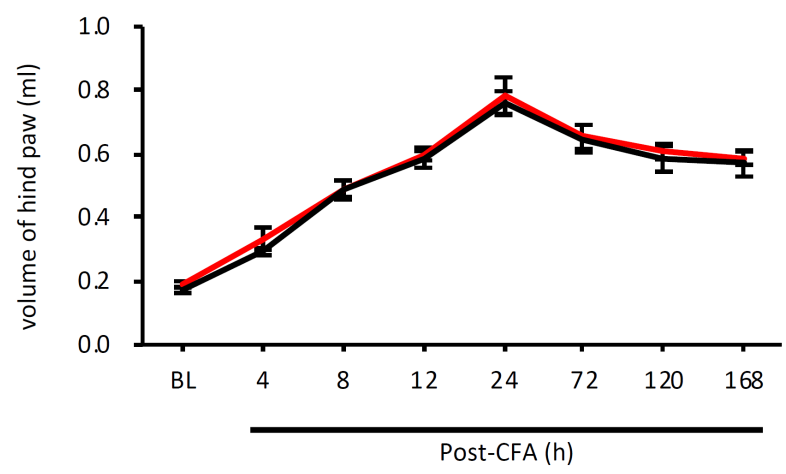

Extended Data Fig. 4 Paw edema after CFA injection. The paw volume in KO mice was comparable to that in WT mice. The time course of paw volume after CFA in KO mice injection was also comparable to that in WT mice. KO mice, $\mathrm{n}=3$ and WT mice, $\mathrm{n}=4$. $\mathrm{BL}$, baseline value (value before CFA injection). $P=$ 0.5885; one-way ANOVA. 


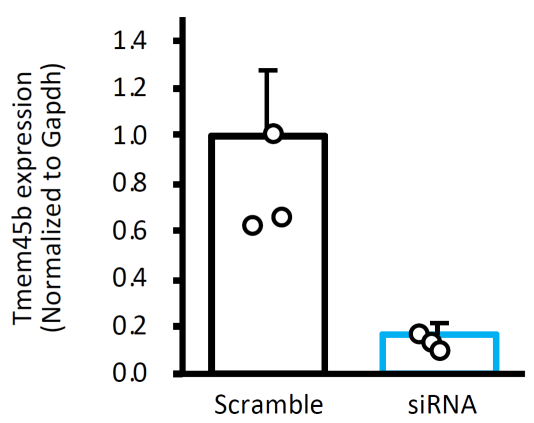

Extended Data Fig. 5 Knockdown efficacy of siRNA against Tmem45b. Knockdown efficacy of siRNA against Tmem $45 b$ used in this study was examined using Lewis lung carcinoma cells, which constitutively expressed Tmem45b. A bar shows mean of relative expression level of Tmem $45 \mathrm{~b}$ mRNA normalized by Gapdh obtained from 3 experiments. $P=0.001$; two-tailed unpaired Student's $t$ test. 
Supplementary Table 1. Sequences of gRNA

TCAAGGGCCATGCTCTCCCAGTTTTAGAGCTAGAAATAGCAAG

Tmem45b gRNA1 TTAAAATAAGGCTAGTCCGTTATCAACTTGAAAAAGTGGCACCG AGTCGGTGCTTTT

TAGGTCAAGGAGTAGCATGGGTTTTAGAGCTAGAAATAGCAAGT

Tmem45b gRNA2 TAAAATAAGGCTAGTCCGTTATCAACTTGAAAAAGTGGCACCGA GTCGGTGCTTTT 
Supplementary Table 2. Sequences of primers used in this study

\begin{tabular}{ll}
\hline Tmem45b genotyping (Tmem45b-1) & GAGAAGTCCTGTGTTAGAGTAAGGTGG \\
Tmem45b genotyping (Tmem45b-2) & CTAGCAGTCATCTAGACCCTGTCCTAT \\
Tmem45b 5'deletion sequence & TGAGGTTGTCCTCCAGCCT \\
Tmem45b 3'deletion sequence & ACCAGAAGCCCTATCACACA \\
Tmem45b cRNA probe-forward & ATGGCAAACTTCAAGGGCCATGCT \\
Tmem45b cRNA probe-reverse & TCACTCCTCATCCGAGCCACTCAAAAG \\
Tmem45b qPCR-forward & GCAATCATTGGGATCCTGGCA \\
Tmem45b qPCR-reverse & AGGGGCACGATGTGGAAGTA \\
Gapdh qPCR-forward & GGGTGTGAACCACGAGAAAT \\
Gapdh qPCR-reverse & ACTGTGGTCATGAGCCCTTC \\
\hline
\end{tabular}


Supplementary Table 3. Colocalization of Tmem45b with IB4, CGRP, TRPV1 and NF200 in DRG neurons.

\begin{tabular}{l|c} 
Marker & Number of double positive neurons / number of each marker positive neurons \\
\hline IB4 & $1174.5 \pm 92.8 / 1256.8 \pm 70.5$ \\
CGRP & $96.0 \pm 33.5 / 1368.5 \pm 113.1$ \\
TRPV1 & $260.8 \pm 63.0 / 1408.5 \pm 486.2$ \\
NF200 & $2.3 \pm 2.6 / 1443.0 \pm 214.5$ \\
\hline
\end{tabular}

Seven to 8 slices of L4/5 DRGs per mouse were analyzed. Data from 4 mice were averaged. 


\section{Supplementary Files}

This is a list of supplementary files associated with this preprint. Click to download.

- nreditorialpolicychecklist.pdf

- nrreportingsummary.pdf 